\title{
Influence of bulk dielectric polarization upon PD transients
}

\author{
Pedersen, Aage; Crichton, George C; McAllister, lain Wilson
}

Published in:

Proceedings of the Conference on Electrical Insulation and Dielectric Phenomena

Link to article, DOI:

10.1109/CEIDP.1995.483729

Publication date:

1995

Document Version

Publisher's PDF, also known as Version of record

Link back to DTU Orbit

Citation (APA):

Pedersen, A., Crichton, G. C., \& McAllister, I. W. (1995). Influence of bulk dielectric polarization upon PD transients. In Proceedings of the Conference on Electrical Insulation and Dielectric Phenomena (pp. 323-326). IEEE. https://doi.org/10.1109/CEIDP.1995.483729

\section{General rights}

Copyright and moral rights for the publications made accessible in the public portal are retained by the authors and/or other copyright owners and it is a condition of accessing publications that users recognise and abide by the legal requirements associated with these rights.

- Users may download and print one copy of any publication from the public portal for the purpose of private study or research.

- You may not further distribute the material or use it for any profit-making activity or commercial gain

- You may freely distribute the URL identifying the publication in the public portal 


\title{
Influence of Bulk Dielectric Polarization upon PD Transients
}

\author{
A. Pedersen G.C. Crichton and I.W. McAllister \\ Physics Department Electric Power Engineering Department \\ The Technical University of Denmark \\ 2800 Lyngby \\ DENMARK
}

\begin{abstract}
From a field-theoretical approach, it has been possible to develop a physically valid theory of partial discharge transients. The theory is based upon the concept of the charge induced upon the detecting electrode by the partial discharge. This induced charge is shown to be composed of a component associated with the actual space charge in the void, and one related to changes in the bulk polarization brought about by changes in the field external to the void due to this space charge. The magnitude of the induced charge and its components are discussed in relation to a heterogeneous bulk dielectric system.
\end{abstract}

\section{Introduction}

The charge induced by a partial discharge on the the detecting electrode can be evaluated using either the $\lambda$-function, or the $\phi$-function $[1,2]$. These functions take account of the dielectric polarization either implicitly $(\lambda)$ or explicitly $(\phi)$. Hence by using the two functions it becomes possible toidentify the influence of the dielectric polarization upon the induced charge. This influence is associated with the change in polarization $\delta \vec{P}$ due to the change in the electric field $\delta \vec{E}$ arising from the partial discharge space charge.

In the present paper, the influence of the bulk dielectric geometry upon $\delta \vec{P}$ is examined for a two dielectric system.
It is shown that the component of the induced charge due to $\delta \vec{P}$ may increase or decrease depending upon the ratio of the dielectric permittivities and within which medium the void is located. This increase/decrease is of course reflected in the Poissonian induced charge $q$.

\section{The Poissonian Induced Charge}

The induced charge can be described in terms of a Poissonian and a Laplacian component [3]. The Poissonian induced charge is that component of the induced charge which is rigidly linked to the space charge source, and which together with this source gives rise to the Basic Poisson Field [2]. Mathematically, the final value of the Poissonian induced charge $q$, due to a partial discharge, can be resolved into two components:

$q=q_{\mu}+q_{P}$

where $q_{\mu}$ is the induced charge directly associated with the space charge in the void, and $q_{P}$ represents the induced charge related to the change in dielectric polarization $(\delta \vec{P})$ due to the presence of this space charge [2]. With reference to induced charge, the effect of the void wall charges can be considered as the effect of an electric dipole of moment $\vec{\mu}$ located within the void [1]. The Poissonian induced charge arising from a dipole is given by

$q=-\vec{\mu} \cdot \vec{\nabla} \lambda$

where $\lambda$ represents the proportionality 
factor between the charge in the void and the induced charge on the detecting electrode. The $\lambda$-function is a solution of the general Laplace equation [2]

$\vec{\nabla} \cdot(\varepsilon \vec{\nabla} \lambda)=0$

in which $\varepsilon$ denotes permittivity. The boundary conditions are $\lambda=1$ at the detecting electrode, and $\lambda=0$ at the surfaces of all other electrodes. In addition, the following condition must be fulfilled at all dielectric interfaces such as the walls of voids

$\varepsilon_{+}(\partial \lambda / \partial n)_{+}=\varepsilon_{-}(\partial \lambda / \partial n)_{-}$

where $\lambda$ is differentiated in the direction normal to the interface, and the signs + and - refer to each side of the interface, respectively. Any method of solving Laplace's equation can be used to determine $\lambda$.

If, however, the dimensions of the void are such that $\vec{\nabla} \lambda$ may be assumed constant within the void, then we can introduce another function, $\lambda_{0}$, which, in the absence of the void, represents the unperturbed $\lambda$-function. As $\lambda$ is a solution of Laplace's equation, then by mathematical analogywith electrostatic fields, the relationship between the $\lambda$ and $\lambda_{0}$ functions is given by

$\vec{\nabla} \lambda=h \vec{\nabla} \lambda_{0}$

For the voids under consideration, the parameter $h$ is a scalar which depends on the void geometry and the relative permittivity of the bulk medium. Following the introduction of $\lambda_{0}$, the Poissonian induced charge on the detecting electrode may be expressed as

$q=-\overrightarrow{h \mu} \cdot \vec{\nabla} \lambda_{0}$

The component of the Poissonian induced charge related to the void space charge alone may be obtained from $q_{\mu}=-\vec{\mu} \cdot \vec{\nabla} \phi$

where $\phi$, another proportionality factor, is a solution of the reduced Laplace equation [2]

$\nabla^{2} \phi=0$

The boundary conditions are $\phi=1$ at the detecting electrode, and $\phi=0$ at the surfaces of all other electrodes. Hence, from (1), (6) and (7), the polarization component $q_{p}$ of the Poissonian induced charge may be expressed as

$q_{P}=-\vec{\mu} \cdot\left(h \vec{\nabla} \lambda_{0}-\vec{\nabla} \phi\right)$

The $\lambda_{0}$ Function

For a homogeneous dielectric system, $\lambda_{0}$ is a solution of the reduced Laplace equation, and hence in such situations $\lambda_{0}$ and $\phi$ are synonomous. Consequently (9) would reduce to

$q_{P}=-(h-1) \vec{\mu} \cdot \vec{\nabla} \lambda_{0}$

In practice, nearly all dielectric insulation systems are heterogeneous. However, for a very restricted class of dielectric geometries, e.g. a coaxial disc spacer, $\lambda_{0}$ is also a solution of the reduced Laplace equation, and thus $\lambda_{0}$ and $\phi$ are again synonomous. The behaviour of $q_{p}$ in this case has been studied in [ $\left.{ }^{2}\right]$.

To examine a situation in which $\lambda_{0}$ is a solution of the general Laplace equation, we consider a planar electrode geometry with a two layer dielectric. If in rectangular coordinates, the electrodes are represented by $z=0$ and $z=d$, then the dielectric interface is taken as $z=s$, with $s<d$. The permittivity of the upper dielectric is $\varepsilon_{2}$ for which $s \leq z \leq d$, while that of the lower is $\varepsilon_{1}$, for which $0 \leq z \leq s$. 
If the lower electrode is used as the detecting electrode, then the boundary conditions for the $\lambda$-function are $\lambda=1$ for $z=0$ and $\lambda=0$ for $z=d$. Hence the $\lambda$ functions of the two media are given by

$\lambda_{01}=\frac{\varepsilon_{1}(d-s)+\varepsilon_{2}(s-z)}{\varepsilon_{1}(d-s)+\varepsilon_{2} s}$

for $0 \leq z \leq s$, and

$\lambda_{02}=\frac{\varepsilon_{1}(d-z)}{\varepsilon_{1}(d-s)+\varepsilon_{2} s}$

for $s \leq z \leq d$, where the $\lambda_{0}$ subscripts, $1 \& 2$, refer to the lower and upper regions, respectively.

On differentiating with respect to $z$, we obtain the relevant expressions for the associated $\lambda$-gradients :

$\vec{\nabla} \lambda_{01}=\frac{-\varepsilon_{2} \vec{e}}{\varepsilon_{1}(d-s)+\varepsilon_{2} s}$

and

$\vec{\nabla} \lambda_{02}=\frac{-\varepsilon_{1} \vec{e}}{\varepsilon_{1}(d-s)+\varepsilon_{2} s}$

where $\vec{e}$ is a unit vector in the positive $z$ direction.

For a homogeneous medium, $\lambda_{0}=\phi$ and thus for a planar system we have

$\vec{\nabla} \lambda_{0}=\vec{\nabla} \phi=-\frac{\vec{e}}{d}$

Both (13) and (14) reduce to this expression for $\varepsilon_{1}=\varepsilon_{2}$.

Heterogeneous Dielectric System

To undertake a comparative as ses sment of the influence of the dielectric geometry upon PD transients we will assume that the dipole $\vec{\mu}$, associated with the charge which accumulates at the void wall following a partial discharge, is considered a constant in this study. Furthermore, it will be as sumed that the void is more than 10 times its greatest linear dimension from the dielectric interface, such that the $\vec{\nabla} \lambda$ distribution within the void is effectively uniform: i.e. the existence of the interface does not perturb $\vec{\nabla} \lambda$ in the void. This assumption implies that the concept of $h$ is valid and that (5) may be employed.

Variation of $q_{P n}$

With respect to the component of the induced charge related to $\delta \vec{P}$, we have upon combining (9) and (6)

$\frac{q_{P n}}{q_{n}}=\frac{-\vec{\mu} \cdot\left(h_{n} \vec{\nabla} \lambda_{o n}-\vec{\nabla} \phi\right)}{-\vec{\mu} \cdot h_{n} \vec{\nabla} \lambda_{o n}}$

where $q_{n}$ is the Poissonian induced charge of the heterogeneous system, with $n=1,2$ depending in which dielectric medium the void is located.

On account of the planar geometry, we may regard the dipole moment as being directed away from the coordina te origin. This moment can then be expressed as

$\vec{\mu}=\mu \vec{e}$

On performing the vector operations, (16) simplifies to give

$\frac{q_{p n}}{q_{n}}=1-\frac{\mathrm{d} \phi / \mathrm{d} z}{h_{n} \mathrm{~d} \lambda_{0 n} / \mathrm{d} z}$

Hence using (13), (14) and (15) we obtain for a void in medium 1

$\frac{q_{P 1}}{q_{1}}=1-\frac{\varepsilon_{1}(d-s)+\varepsilon_{2} s}{\varepsilon_{2} h_{1} d}$

and for medium 2

$\frac{q_{P 2}}{q_{2}}=1-\frac{\varepsilon_{1}(d-s)+\varepsilon_{2} s}{\varepsilon_{1} h_{2} d}$ 
As both (19) and (20) contain $h$, this implies that $q_{p n}$ is dependent upon the void geometry. In this study we will assume the void to be spherical, in which case we have

$h=\frac{3 \varepsilon_{r}}{1+2 \varepsilon_{r}}$

where $\varepsilon_{r}$ is the relative permittivity of the dielectric containing the void.

The variation of $q_{P n} / q_{n}$ with $\varepsilon_{2} / \varepsilon_{1}$ is shown in Fig.1 for $\varepsilon_{r}=4$ and several values of $s / d$. From the figure it is seen that $q_{P_{n}}$ is increased when the void is located in the medium of lower permittivity. Likewise a decrease occurs when located in the medium of higher permittivity. This reduction can be of such a degree that the polarity of $q_{P D}$ is reversed.

When the void is in the lower medium, the nearer the dielectric interface is to the detecting electrode, the greater are the relative changes in these induced charges. The opposite behaviour occurs when the void is in the upper medium; i.e., the further the interface is from the detecting electrode, the greater is the effect.

Owing to the simple geometry under discussion, the behaviour of $q_{P n}$ is symmetrical with respect to $s / d$ and $(1-s / d)$.

For a fixed void location $q_{\mu}$ is dependent only on $\vec{\mu}$, which we assumed constant. Hence variations in $q_{p}$ will be directly reflected in the magnitude of the Poissonian induced charge $q$, see (1).

\section{Conclusion}

It is demonstrated that changes in dielectric polarization arising from a partial discharge can significantly affect the magnitude of the Poissonian induced charge. For a two dielectric system, this influence is dependent upon the ratio of the dielectric permittivities and within which medium the void is located. Hence the interpretation of partial discharge transients in terms of discharge phenomena within the void is even more complicated when heterogeneous systems are involved.

\section{References}

[1] A. Pedersen, G.C. Crichton and I.W. McAllister, "The Theory and Measurement of Partial Discharge Transients", IEEE Trans. Elect. Insul.,Vol.26, pp.487-497, 1991.

[2] A. Pedersen, G.C. Crichton and I.W. McAllister, "The Functional Relationship Between Partial Discharges and Induced Charge", submitted to IEEE Trans. Diel. \& Elect. Insul., 1994.

[3] A. Pedersen, G.C. Crichton and I.W. McAllister, "Partial Discharge Detection: Theoretical and Practical Aspects", IEE Proc.- Sci. Meas. Technol., Vol.142 pp.29-36, 1995.

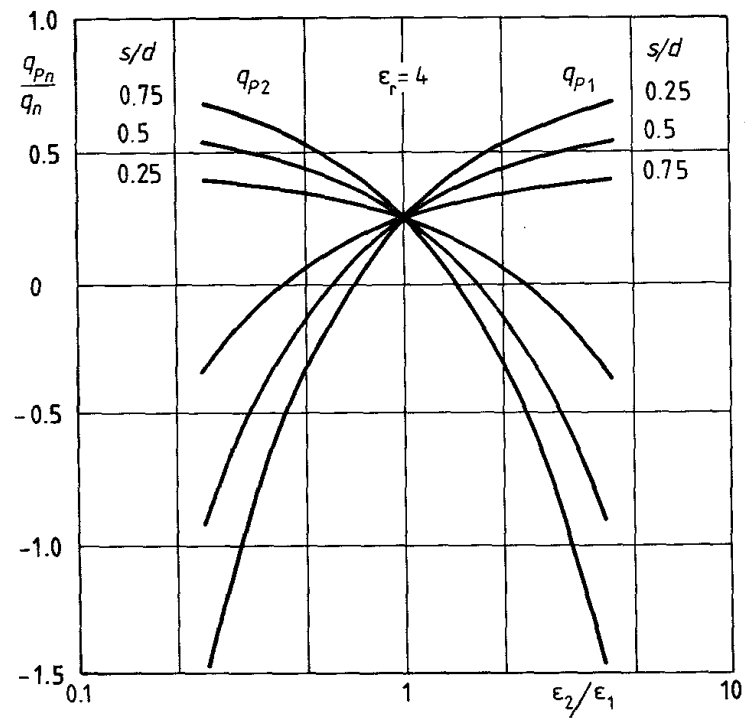

Fig. I Variation of the polarization component of the Poissonian induced charge. 